\title{
MELT-MEDIATED LASER CRYSTALLIZATION OF THIN FILM NITI SHAPE MEMORY ALLOYS
}

\author{
Paper (M406)
}

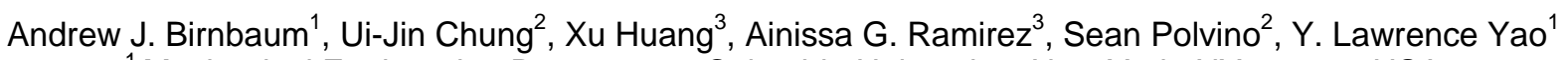 \\ ${ }^{1}$ Mechanical Engineering Department, Columbia University, New York, NY, 10027, USA \\ ${ }^{2}$ Program in Materials Science, Department of Applied Physics and Applied Mathematics, Columbia \\ University, New York, NY, 10027, USA \\ ${ }^{3}$ Mechanical Engineering Department, Yale University, New Haven, CT, 06511, USA
}

\begin{abstract}
This work utilizes pulsed, melt-mediated laser crystallization techniques to control the spatial distribution of crystalline zones within an as sputterdeposited amorphous matrix. Since shape memory responses stem from crystallographic shifts, only the selectively crystallized regions exhibit these properties. This process provides not only spatial control over the shape memory response, but potentially, through proper use of operational parameters, the shape memory response itself, i.e. phase transformation temperature, transformation strain, recovery stress etc. The solidification process is monitored in situ via transient reflectance. Furthermore, the effects of varying energy density within the irradiated region are examined with respect to the resulting micro-structure via atomic force microscopy (AFM), electron backscatter diffraction (EBSD) and x-ray diffraction (XRD).
\end{abstract}

\section{Introduction}

Pulsed, melt mediated crystallization techniques have been studied extensively for a range of material classes including semiconductors, elemental and alloyed metals [1-3]. The process provides a flexible means for spatial control over crystalline/amorphous regions as well as very precise control over the resulting microstructure itself. By varying the incident laser energy density, energy distribution within the irradiated area, beam shape and patterned geometries, grain size and shape as well as texture may be specifically tailored toward a range of applications. Silicon thin films have been successfully processed in this manner for the production of thin film transistors (TFT's) for use in active matrix displays (e.g. LCD and OLED) as well as the active medium in thin film solar cells [4]. These applications also rely on the ability of the process for controlling the density of microstructural defects (e.g. high-angle grain boundaries) which have significant effects on device performance[5]. Spaepen, Turnbull and Lin [6-8] have used the pulsed melt mediated process to produce and study the behaviour of thin film metallic glasses and thin film metallic alloys. This process is well suited toward this end owing to exceedingly high quench rates [7]. Interest in processing elemental metals has also increased as a means for producing and tailoring microstructures for interconnects in IC circuitry [9].

Pulsed, melt-mediated laser crystallization techniques have yet to be utilized in processing thin film shape memory alloys and hold great potential for control over the resulting structure. Although shape memory alloys' (SMA's) non-conventional properties have been studied extensively over the last four decades, their actual implementation has been limited due to several complicating factors. A main impediment to application, particularly for thermally induced macro-scale actuation applications is poor dynamic response stemming from long heat dissipation times. Recently, thin film shape memory structures have received increasing attention for micro-scale actuation as they do not suffer from this limitation due to their exceedingly low thermal mass. The vast majority of this work has been limited to thin films whose shape memory properties are homogenous throughout the film. Ishida et. al. [10-12] conducted a series of in depth investigations on the effects of furnace, solid phase crystallization parameters (i.e. annealing temperature and dwell time) on the resulting shape memory properties of NiTi sputtered thin films, while Gil [13] directly examined the effect of grain size on martensitic phase transformation temperatures. Lee and Ramirez [14,15] have recently conducted a series of investigations into the kinetics of the solid phase crystallization process via in situ TEM observations. A wide variety of proposed SMA based actuation devices may be found in [16-19], again, all relying on homogenous shape memory properties. 
Uniform Energy Density (Flood Irradiation)

NiTi

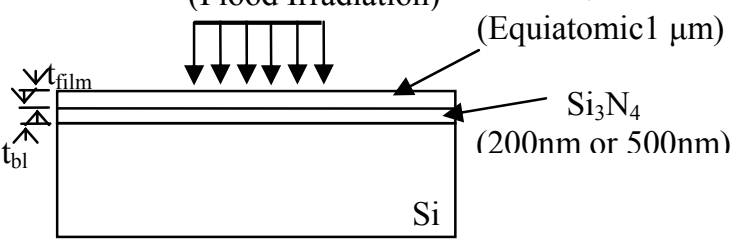

Figure 1: $1 \mu \mathrm{m}$, equiatomic NiTi film sputtered at room temperature on $200 \mathrm{~nm}$ or $500 \mathrm{~nm} \mathrm{Si}_{3} \mathrm{~N}_{4}$ barrier layer on $\mathrm{Si}$ (100) substrate.

Several investigations on the use of laser annealing in order to control the spatial extents of crystalline regions have been conducted, although they have all been restricted to $\mathrm{CW}$, solid phase processing. Bellouard et. al. [20,21] used a near IR CW laser to selectively solid-phase crystallize a sputter deposited NiTi thin film for the purposes of fabricating a microgripper whose only active component was the crystallized portion of the device. He et. al. [22] have investigated the use of a $\mathrm{CW} \mathrm{CO}_{2}$ laser to selectively anneal a NiTi thin film via solid-phase crystallization as well.

This work proposes the use of pulsed, meltmediated laser crystallization techniques to control the spatial distribution of crystalline zones within an amorphous matrix. Since shape memory responses stem from crystallographic shifts, only the selectively crystallized regions will exhibit these properties. This process provides not only spatial control over the shape memory response, but through proper use of operational parameters, has the potential for tailoring the shape memory response itself, i.e. phase transformation temperature, transformation strain, recovery stress etc. The use of a melt-mediated process also provides several advantages over homogenous furnace annealing as well as CW, solid phase techniques. These include increased efficiency for large scale fabrication due to single shot batch processing capabilities (i.e. no rastering), increased control over the resulting microstructure, and features with sharp boundaries due to low pulse duration to thermal diffusivity time scales. Crystallization rates for the pulsed process ( $\sim$ nanosecond pulse durations) also far exceed those of CW processing whose scan speeds are on the order of $3-5 \mathrm{~mm} / \mathrm{s}$ and furnace annealing times ranging from tens of seconds to several hours. The pulsed process is also advantageous as it may be performed on device films in situ in fairly close proximity to other possibly thermally sensitive components.

\section{Experimental Setup}

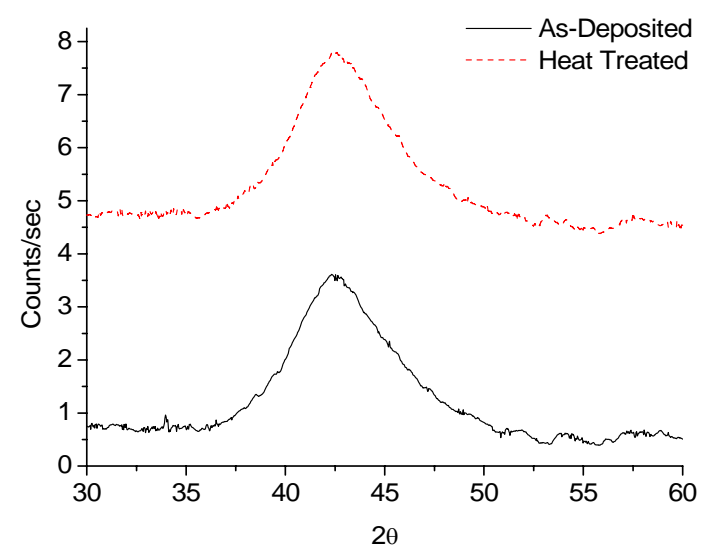

Figure 2: X-ray diffraction spectrum of asdeposited film. Note the amorphous scattered signal indicative of room temperature sputtered NiTi. Heat treated spectrum shifted for clarity.

Near equiatomic, $1 \mu \mathrm{m}$ NiTi films were deposited by simultaneous co-sputtering from an alloyed NiTi target and pure titanium target at powers of 302 and 50 watts respectively for 1400 seconds at an argon pressure of 3 mTorr. The films were deposited on (100) silicon wafers on top of 500nm of PECVD silicon nitride $\left(\mathrm{Si}_{3} \mathrm{~N}_{4}\right)$. The specimen configuration may be seen in Figure 1. The deposition was performed at room temperature, and thus resulted in an amorphous film as confirmed by x-ray diffraction (XRD), as seen in Figure 2. Furthermore, the film composition as near 50 at.\% $\mathrm{Ti}$ was confirmed via a calibrated electron microprobe.

Prior to laser processing, it was also necessary to subject the film to a pre-heat treatment in order to thermally oxidize the film surface to enhance optical absorption at the laser wavelength. Further details of the oxidation process may be found in the discussion below. Lee et. al. [14] suggests that solid phase crystallization of the film will not occur for the above heat treatment parameters. However, as a precaution, XRD (see Fig. 2) was conducted post-heat treat confirming the film remained amorphous. Films were then single shot, pulse irradiated by a $308 \mathrm{~nm}$ wavelength, $\mathrm{XeCl}$ excimer laser with 30 ns pulse duration over a wide range of incident laser energy densities. Energy density was uniform within the irradiated region whose geometry was square, $320 X 320 \mu \mathrm{m}$. The laser system is synchronized with the underlying $\mathrm{XYZ}$ motion system such that an array of energy densities may be applied over a spatial array on a single specimen. An array of irradiated regions may be seen in Figure 3. Each square region is an irradiated area of a single uniform energy density with $10 \mu \mathrm{m}$ spacing. The system is also equipped to 


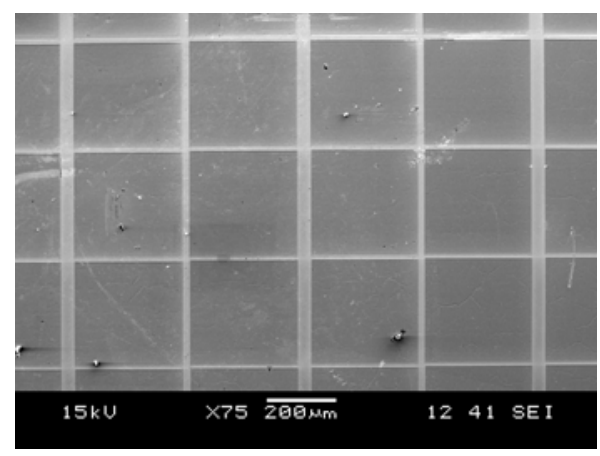

Figure 3: SEM image of an array of irradiated regions. Irradiated regions are $320 \times 320 \mu \mathrm{m}$ with uniform energy density. Each square region represents one energy density.

perform in situ transient reflectance analyses through the use of a laser diode (670 $\mathrm{nm}$ wavelength) and photodiode to real time monitor the surface reflectance of the specimen during irradiation.

\section{Theoretical Background}

The underlying phenomena governing pulsed laser crystallization have their basis in highly nonequilibrium heat flow and solidification. The laser energy is coupled to the film via non-linear absorption over, in this case a metallic matrix, a shallow near surface layer resulting in melting and resolidification. The extent of the melted region is determined by the incident laser energy, the optical and thermo-physical properties of the material and the heat of fusion. Solidification is accompanied by a release of energy in the form of the latent heat of fusion, $\mathrm{H}_{\mathrm{f}}$ at the solid liquid interface which further contributes to the thermal profile within the film. The transient temperature distribution is therefore governed by the classical heat equation:

$$
\begin{aligned}
& \rho^{i}\left(\Phi, T^{i}\right) c_{p}^{i}\left(\Phi, T^{i}\right) \frac{\partial T^{i}}{\partial t}=\nabla\left(k^{i}(\Phi, T) \nabla T^{i}\right)+. . \\
& . .+\Sigma Q_{n u c}(x, y, z, t)+Q_{\text {laser }}(x, y, z, \lambda)
\end{aligned}
$$

where $T^{i}, \rho^{i}, c_{p}^{i}$ and $k^{i}$ are the temperature, temperature dependent density, specific heat and thermal conductivity, respectively for the $i^{\text {th }}$ material and phase, $\Phi$. $Q_{n u c}(x, y, z, t)$ are the energy contributions from latent heat of solidification and $Q_{\text {laser }}(x, y, z, \lambda)$ is the laser energy input that is actually coupled to the film, taking the optical properties of the material into account. Convection to the surroundings is not taken to account due to the time scale over which solidification occurs. It is also assumed that radiation is negligible compared with heat conduction through the film.

Resolidification of the film may proceed via several distinct, highly non-equilibrium pathways. Crystallization, which is the focus of this work, may proceed via epitaxial re-growth, heterogeneous nucleation and growth or homogeneous nucleation and growth. Nucleation, homogeneous or heterogeneous, occurs via the formation of "solid" embryos at temperatures far below the equilibrium melting temperature of the material due to a decrease in the system's Gibbs free energy. Classical nucleation theory (CNT) models this phenomenon by assuming, in the case of homogeneous nucleation, the formation of a spherical embryo of radius, $r$ and for heterogeneous, a spherical cap of radius $r$ in contact with a flat catalytic surface at a contact angle, $\theta$, the difference in Gibbs free energy reaches some critical threshold. The change in Gibbs free energy (homogeneous) goes according to [23]:

$$
\Delta G^{t o t}=\frac{4 \pi}{3} r^{3} \Delta G_{v}+4 \pi r^{2} \sigma_{s}
$$

where $\Delta G_{v}$ is the difference in volumetric free energy between the liquid and solid (a negative quantity below the equilibrium melting temperature) and $\sigma_{s}$ is the surface free energy per unit area (positive value) associated with the formation of a new solid/liquid interface for a spherical embryo. A critical size nucleus, $r^{*}$, is formed when the radius is large enough such that a further increase in size results in a decrease in the total Gibbs free energy. For homogeneous nucleation:

$$
r^{*}=\frac{2 \sigma_{s}}{\Delta G_{v}}, \Delta G_{\mathrm{hom}}^{*}=\frac{16 \pi \sigma_{s}^{3}}{3 \Delta G_{v}^{2}}
$$

The homogeneous nucleation rate per unit volume at a given undercooling is therefore:

$$
I_{\text {hom }}=I_{0, \text { hom }} \operatorname{Exp}\left(\frac{-\Delta G_{v}^{*}}{k T}\right)=I_{0, \operatorname{hom}} \operatorname{Exp}\left(\frac{-16 \pi \sigma_{s}^{3}}{3 k T \Delta G_{v}^{2}}\right)
$$

For heterogeneous the details of the derivation are omitted but proceed similarly and are given as:

$$
r^{*}=\frac{2 \sigma_{s}}{\Delta G_{v}}, \Delta G_{\text {het }}^{*}=\Delta G_{\mathrm{hom}}^{*} S(\theta)=\frac{16 \pi \sigma_{s}^{3}}{3 \Delta G_{v}^{2}} S(\theta)
$$




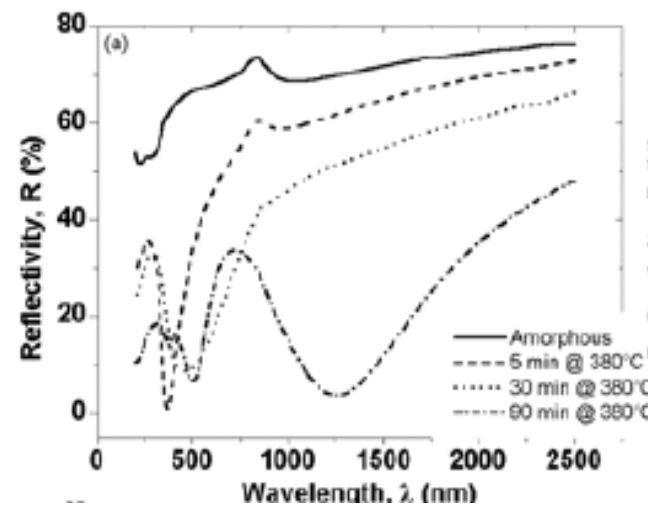

Figure 4: Reflectivity spectrum for an amorphous and a number of heat treated films. This change in reflectivity, due to oxidation, particularly at $\lambda=308 \mathrm{~nm}$ is consistent with observed enhanced laser-film energy coupling [25].

where $S(\theta)$ is a geometric factor taking the contact angle of the nucleus with the catalytic surface, $\mathrm{k}$ is the Boltzmann constant, $I_{0, \text { hom }}$ is a fairly complex kinetic factor related to the frequency with which "liquid" atoms attach to the embryo and the exponential is the number of critical size embryos per unit volume. It should be noted that $S(\theta) \leq 1$ and thus $\Delta G_{\text {het }}^{*} \leq \Delta G_{\text {hom }}^{*}$ Physically, this translates to a lower energy threshold to overcome for nucleation in the heterogeneous case, and in fact most metallic melts are dominated by heterogeneous nucleation [5].

Upon successful nucleation, nuclei will immediately begin to grow. While nucleation requires significant undercooling, growth can occur at fractions of a degree below the equilibrium melting temperature [23]. Growth velocity is a strong function of temperature and is captured by the interface response function which relates the solid/liquid interface velocity with the interface temperature. For pure metallic melts, Turnbull suggests a collision limited theory resulting in:

$$
v_{i}=f v_{s}\left(1-\operatorname{Exp}\left(\frac{\Delta \bar{G}}{R T_{i}}\right)\right)
$$

where $v_{s}$ is the speed of sound in the melt and $f$ represents the fraction of available site on the solid interface upon which liquid atoms may attach. Aziz [24] suggests a modification to Equation 7 for binary alloys by assuming the driving force $\Delta \bar{G}$ is calculated via a weighted average of the chemical potentials of the constituents with respect to their compositions.

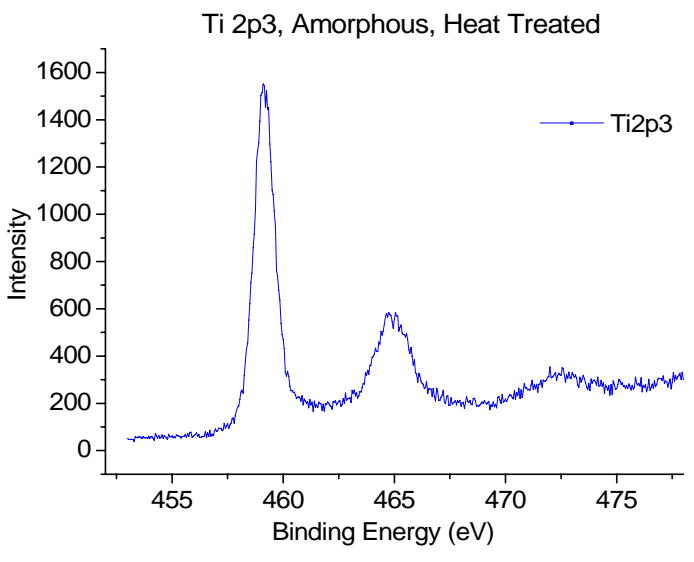

Figure 5: XPS spectrum (Ti2p3) of heat treated film confirming presence of $\mathrm{TiO}_{2}$.

\section{Results and Discussion}

\section{Optical Absorption Enhancement}

Initial attempts at crystallizing an array of films with varying thickness and slight variations in composition over a wide range of energy densities, were unsuccessful, i.e. no microstructure was detectable by either AFM or XRD. Figure 4 is taken from Khelfaoui et. al.'s [25] investigation into the effects of laser induced oxidation of NiTi thin films. It is a plot of optical reflectivity as a function of wavelength for amorphous and oxidized NiTi films. It is clearly seen that optical reflectivity of the amorphous film drops precipitously due to the formation of an oxide after heat treatment at 380 degrees $\mathrm{C}$ for five minutes. This decrease is particularly significant at $308 \mathrm{~nm}$, which corresponds to the wavelength at which the specimen is processed. Thus, it was deduced that initially, most of the incident laser energy was being reflected and most likely only melting a very thin, near surface layer. The growth of a thin oxide resulted in enhanced optical absorption, thus enabling the melting of the film. Figure 5 is a plot of intensity versus binding energy spectrum for the heat treated film obtained via x-ray photoelectron spectroscopy (XPS). It clearly shows the formation of intensity peaks at binding energies consistent with the formation of a layer of $\mathrm{TiO}_{2}$.

\section{II.1 - Partial vs. Complete Film Melting}

At low energy densities, laser crystallization will typically proceed via partial melting [26], i.e. the incident energy density is insufficient for melting the film through the entire thickness. For these cases the surface will melt first and the liquid-solid interface will propagate toward the film/barrier layer interface. At 


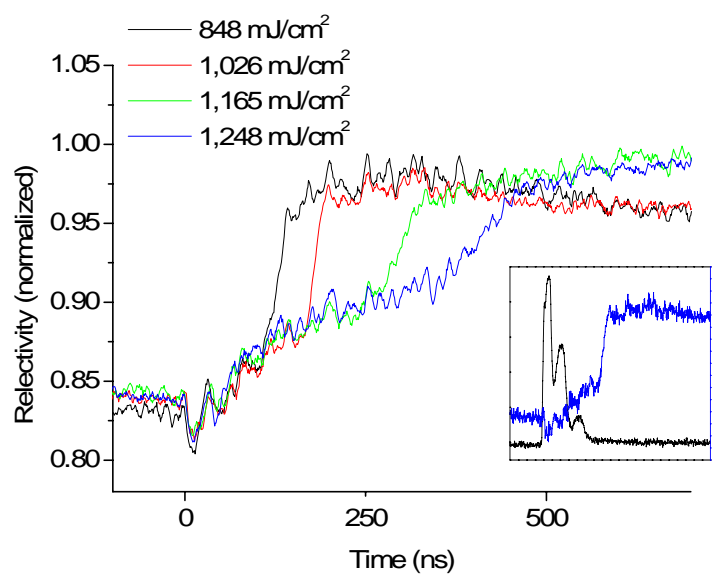

Figure 6: Transient reflectance spectra $(670 \mathrm{~nm})$ for a range of energy densities for $500 \mathrm{~nm}$ silicon nitride barrier layer. Inset shows temporal pulse profile overlaid with representative reflectance spectrum.

some point the interface motion will stop, reverse direction and proceed back toward the film surface. In this regime, maximum grain sizes are typically restricted to the thickness of the film [26].

Upon complete melting, resolidification is delayed due to the requirement of nucleation and therefore deep undercooling. An indicator of complete melting is the appearance of lateral grain growth from the boundary of irradiation. After irradiation, and upon cooling down, lateral growth starts to grow first at or near the equilibrium melting temperature and proceeds perpendicular to the boundary until the interior temperature has decreased sufficiently such that nucleation is triggered. The lateral growth is eventually arrested by impingement with nucleated grains from the interior. Thus lateral growth can only occur if the full thickness of the film has been melted. It should be noted that a third regime typically exists, termed the near complete melting regime [26]. This involves irradiating the film with energy densities slightly lower than the complete melt threshold. This results in local solid "islands" remaining and acting as seeds for growth upon reaching the equilibrium melting temperature during cooling. The regime is typified by exceedingly large grains relative to the film thickness. This regime is not investigated in the current work, but will be examined in future studies.

\section{II.2 Microstructure - 500nm Barrier Layer}

Figure 6 contains representative transient reflectance spectra for a series of energy densities. The surface reflectivity of the irradiated region is continuously monitored by a photodiode receiving a signal from a $670 \mathrm{~nm}$ laser diode. The inset in Figure 6 shows a single reflectance spectrum with the actual
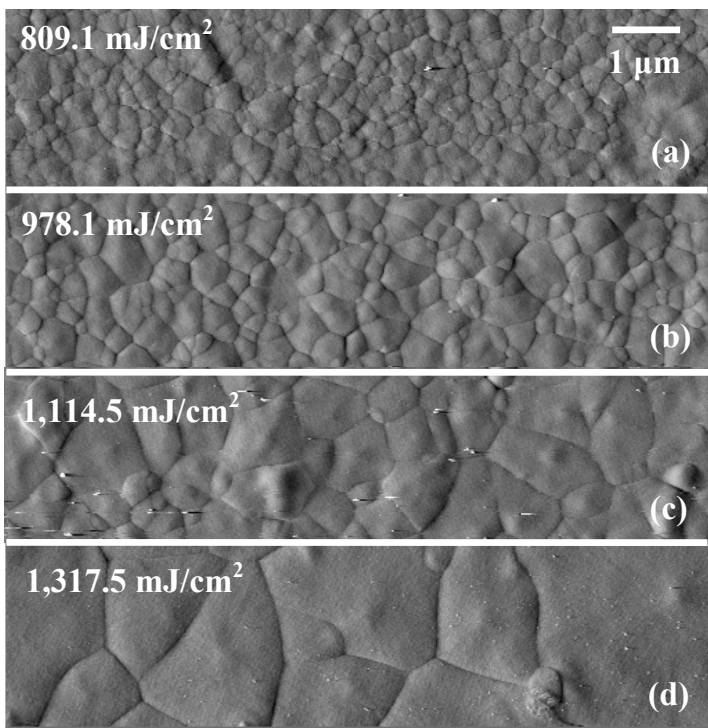

Figure 7 (a-d): AFM images over a range of energy densities for the NiTi film on $500 \mathrm{~nm}$ silicon nitride.

excimer pulse profile overlaid. It is also important to note that as the film is metallic and allows no transmission; only a very shallow near surface layer is providing the reflected signal to the photodiode and thus should be considered to be a truly surface measurement. The pulse is initiated at zero nanoseconds, and the reflectivity values shown in the Figure prior to that represent the surface reflectivity of the amorphous NiTi. Immediately upon irradiation, a sharp drop is seen in the surface reflectivity. This drop is indicative of melting as the molten metal reflects less signal. After dropping, there is a subsequent increase in reflectivity which is most likely showing the temperature dependence of the optical properties of the liquid as the molten surface cools. This is followed by a sudden change in derivative and step-like rise and plateau to a steady higher reflectivity value. This steep increase represents the solidification of the surface, and the new steady state reflectivity is that of the crystalline NiTi. Thus the time elapsed between the initial melting of the film and the advent of the crystalline reflectivity represents the melt duration of the film surface. The change in steady state surface reflectivity is also further evidence of crystallization.

Figures 7(a-d) are AFM images of surface microstructure for a range of energy densities for the NiTi film on $500 \mathrm{~nm}$ of silicon nitride. It is seen that grain size increases monotonically over this energy density range. This is explained by the fact that an increase in energy density results in a higher peak temperature and deeper melt depth and thus a lower overall quench rate. Added time in the molten state 


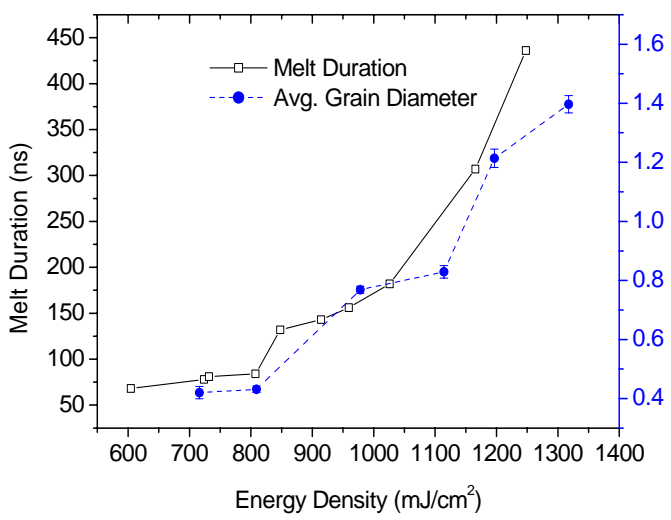

Figure 8: Plot of melt duration (left scale) and average grain size as a function of energy density. Note the monotonic increase for both. Note: Error bars represent standard error.

translates to more time for grain growth. This added time for growth in the molten state is embodied and consistent with the transient reflectance spectra just discussed. Figure 8 clearly shows the functional dependence of melt duration, calculated from the transient reflectance spectra, on energy density with the accompanying increase in average grain size as obtained by EBSD.

Figure 9 shows grain diameter histograms for two representative energy densities. It is seen that at an energy density of $978 \mathrm{~mJ} / \mathrm{cm}^{2}$ the average grain diameter is $770 \mathrm{~nm}$, with a standard deviation of 180 $\mathrm{nm}$. As energy density is increased, the average grain diameter increases, but it is accompanied by an increase in standard deviation as well. For example, an energy density of $1,317 \mathrm{~mJ} / \mathrm{cm}^{2}$ results in an average grain diameter of $1.39 \mu \mathrm{m}$, but a standard deviation of $709 \mathrm{~nm}$. This may be explained by a transition from partial to complete melting. For the partial melting regime, it is assumed that growth initiates from the solid liquid interface and proceeds into the undercooled liquid. As this is an as-deposited amorphous film, the source of these seeds is not yet fully understood. For crystallization of amorphous silicon in the partial melting regime [26], it is assumed that the source of these seeds stems from microcrystalline clusters resulting from the PECVD process. If that assumption is made for this situation, then that would imply that the vast majority of growth events initiate over a very short period of time in the presence of a slightly undercooled liquid and thus the resulting microstructure is fine, but uniform and consistent with the results presented here. Another potential explanation would be heterogeneous nucleation at the solid/liquid interface, however, further investigation is required to confirm.

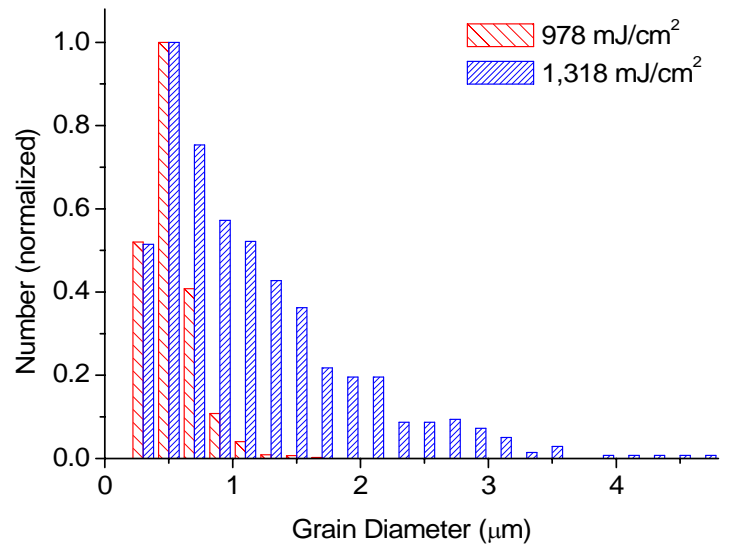

Figure 9: Grain size histograms obtained by EBSD. Note the increase in both average grain size and standard deviation for increases in energy density.

Figures $10(a, b)$ are AFM images from the boundary of irradiation (film on $500 \mathrm{~nm}$ silicon nitride). For the high energy density case $\left(1,317 \mathrm{~mJ} / \mathrm{cm}^{2}\right)$, one observes large, high aspect ratio grains with long axis perpendicular to the irradiated boundary. As previously discussed, the presence of lateral growth at the boundary of irradiation points to the film having undergone complete, through thickness melting. Lateral growth is not present for the low energy density case $\left(978 \mathrm{~mJ} / \mathrm{cm}^{2}\right)$, suggesting that complete melting has not yet occurred, and the transition from partial to complete melting is occurring between the two aforementioned energy densities.

\section{II.3 - Nucleation and Growth}

Heterogeneous and homogeneous nucleation events occur stochastically in space, time and temperature. This results in initial sets of nuclei forming and immediately growing. As growth of primary nuclei proceeds, subsequent nucleation events occur and growth proceeds from those nuclei as well. However, primary nuclei have had much more time for growth before solidification resulting in larger grain sizes, but also much more variation in grain size. Determination of whether solidification proceeds via heterogeneous or homogeneous nucleation is the subject of much debate [5]. However, due to technical complexities due to the exceedingly high rate with which the process occurs, it is very difficult to obtain direct evidence, particularly in metals; due in part to their lack of transparency. One indirect method for determining nucleation pathway is the presence of a preferred texture. Homogeneous nucleation results in grains of varying size and theoretically a totally random texture, as locally, upon formation of an embryo, there are no factors that would lead to a 


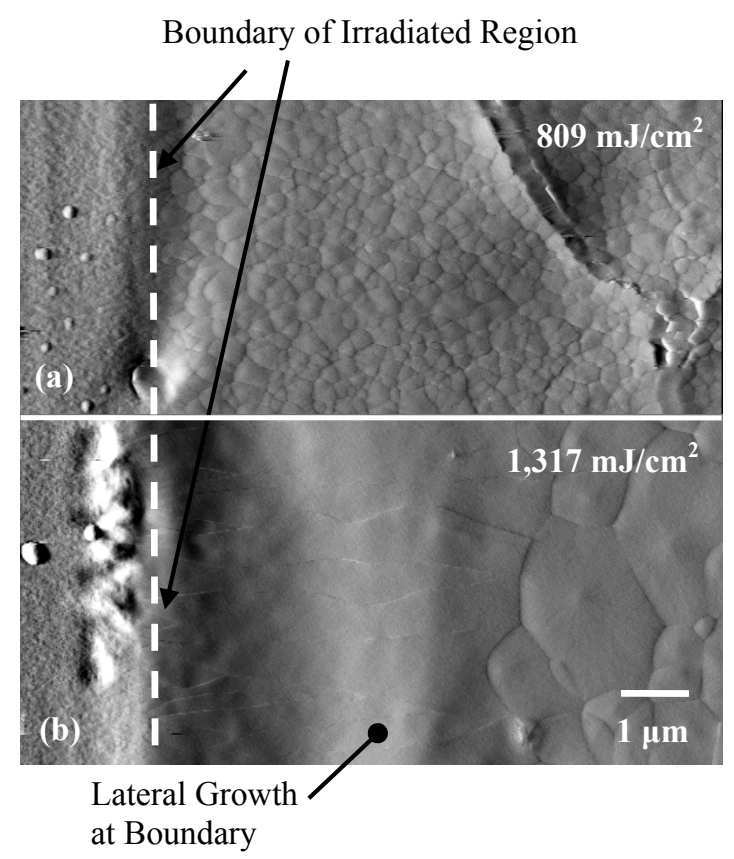

Figure 10: Lateral growth at the boundary begins to appear signaling the transition to complete film melting as energy density goes above 1,300 $\mathrm{mJ} / \mathrm{cm}^{2}$. Note the lack of lateral growth at low energy densities.

preferred orientation. However, heterogeneous nucleation may result in potentially very strong textures. This stems from the energetic properties of the catalytic surface, contact angle and surface tensions of the solid (anisotropic) and liquid media. Certain crystallographic orientations may be energetically favourable over others in terms of their ability to lower the overall energy state.

Figures 11 (a-d) are inverse pole figures obtained over a range of energy densities for the film on $500 \mathrm{~nm}$ silicon nitride. Figure 12a reveals a strong normal texture accompanied by a random in-plane texture for the $978 \mathrm{~mJ} / \mathrm{cm}^{2}$ case. This case is representative over the entire range of energy densities examined. Figures $11(\mathrm{~b}-\mathrm{d})$ are contoured inverse pole figures for $978 \mathrm{~mJ} / \mathrm{cm}^{2}, 1,317 \mathrm{~mJ} / \mathrm{cm}^{2}$ and 1,646 $\mathrm{mJ} / \mathrm{cm}^{2}$ respectively. This persistence in texture may imply that the nucleation process is heterogeneous. However, it is important to note that it persists over both the partial and complete melting ranges. For the partial melting regime there are seemingly two possibilities. If microcrystals do in fact exist, then they have some existing preferred texture due to the deposition process explaining the post process texture preference. The other possibility is that heterogeneous nucleation is occurring at the solid/liquid interface. (a)

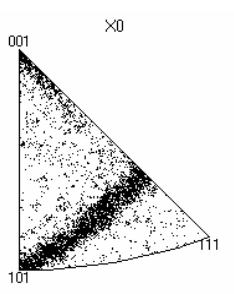

20

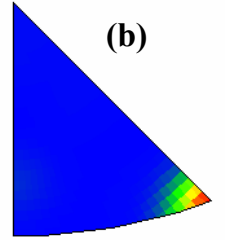

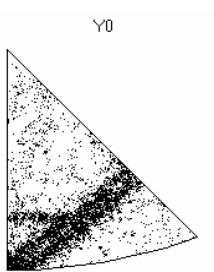

20

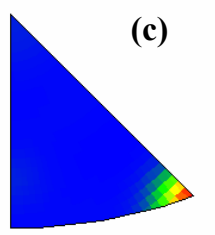

20
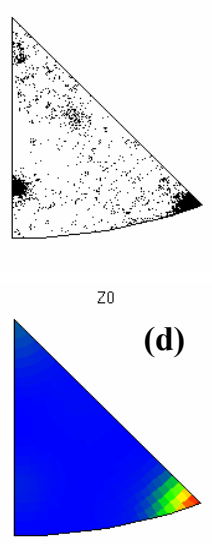

Figure 11 (a-d): (a) Shows inverse pole scatter plots for in $\left(\mathrm{X}_{0}, \mathrm{Y}_{0}\right)$ and normal $\left(\mathrm{Z}_{0}\right)$ orientations for a $978 \mathrm{~mJ} / \mathrm{cm}^{2}$ energy density and is indicative of the entire range of energy densities. It reveals a strong (111) normal texture and random in-plane texture. (b-d) are inverse pole figures for the normal direction for $978 \mathrm{~mJ} / \mathrm{cm}^{2}, 1,317 \mathrm{~mJ} / \mathrm{cm}^{2}$ and $1,646 \mathrm{~mJ} / \mathrm{cm}^{2}$.

Further investigation is required though in order to determine the source of this phenomenon.

\section{III. - The Effect of Barrier Layer Thickness: NiTi Film on 200nm Silicon Nitride}

In addition to applied energy density, many factors can affect the resulting film microstructure. One important consideration is the barrier layer upon which the film is deposited. It acts as an influence on the transient thermal profile as well as a barrier to diffusion between the film and substrate. It typically also has a much higher equilibrium melting temperature than the film being processed so as to inhibit barrier layer melting and diffusion/mixing. The barrier layer composition, crystal structure (or lack thereof) and thickness are major contributors to the crystallization phenomena. Figures 12 (a-d) are AFM images of surface microstructure over a range of energy densities for a film on $200 \mathrm{~nm}$ of silicon nitride. A similar pattern is seen in terms of the monotonic increase in average grain size as well as an increase in the variation in grain size as a function of energy density. Unfortunately, due to limitations in the spatial resolution of the EBSD system, quantitative information on grain statistics are not available. However, it may be observed visually that the maximum sized grains are far smaller than those 


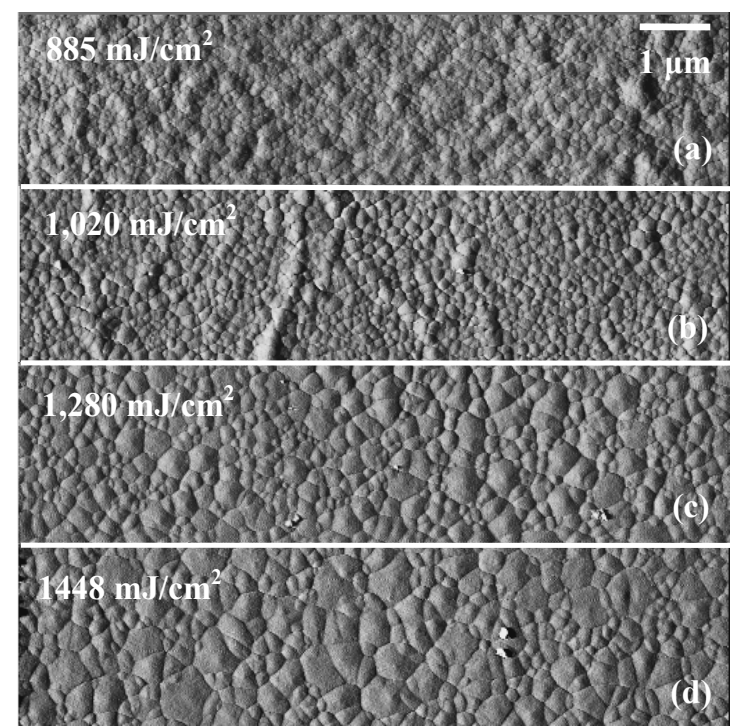

Figure 12(a-d): AFM images over a range of energy densities for the NiTi film on $200 \mathrm{~nm}$ silicon nitride.

discussed above for the NiTi film on $500 \mathrm{~nm}$ silicon nitride. This is explained by the fact that the silicon nitride has a far lower thermal conductivity than the underlying silicon and thus acts as an insulator, impeding the flow of heat. However, when comparing the $200 \mathrm{~nm}$ to the $500 \mathrm{~nm}$ barrier layer, the $500 \mathrm{~nm}$ layer acts as a more efficient insulator and thus maintains the film at molten temperatures longer and thus allows more time for grain growth. Although the microstructures are smaller for the $200 \mathrm{~nm}$ barrier layer case, the crystallization process still proceeds along similar phenomenological paths, i.e. at lower energy densities the film is only partially melted and transitions to complete melting as a process specific threshold is crossed.

Figure 13 is a series of experimentally obtained XRD spectra for the film on $200 \mathrm{~nm}$ of silicon nitride over a range of energy densities. The $\mathrm{x}$ ray penetration depth is far longer than the film thickness. This is confirmed by the presence of diffraction from the silicon substrate (not shown). Therefore the signal obtained stems from diffraction over the entire film thickness. For the low energy density, the emergence of a small peak suggests the formation of crystalline structure. As energy density increases, the peak integrated intensity increases due to larger volumes of crystalline material present. For the same sampling area, the most plausible explanation for this increase is an increase in the depth over which crystalline material exists. The peak integrated intensity does begin to plateau at approximately 1,300 $\mathrm{mJ} / \mathrm{cm}^{2}$, suggesting a transition from partial to

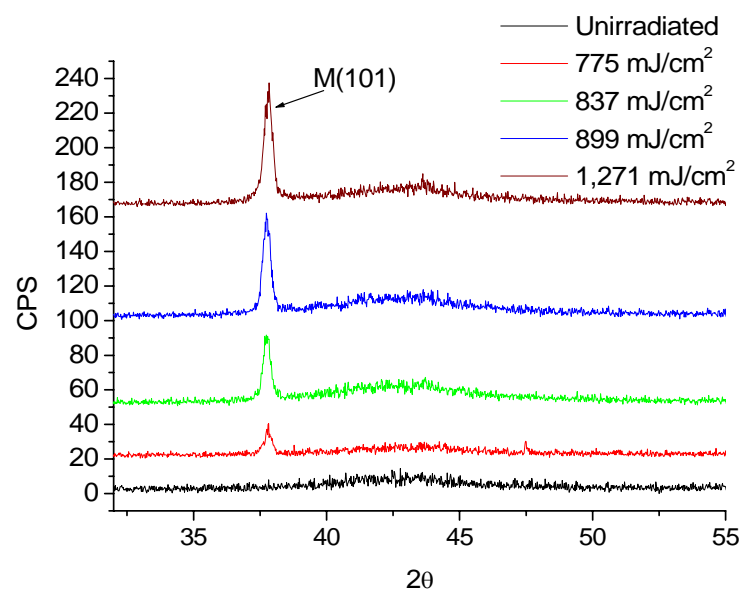

Figure 13: X-ray diffraction spectrum for the $1 \mu \mathrm{m}$ NiTi film on $200 \mathrm{~nm}$ silicon nitride. Note the gradual increase in diffracted intensity as laser energy density is increased.

complete melting. This should be noted as this is a similar complete melting threshold as that of the 500 $\mathrm{nm}$ barrier layer, suggesting that melt depth is fairly insensitive to barrier layer thickness for a $1 \mu \mathrm{m} \mathrm{NiTi}$ film. This may be explained by the fact that the velocity of the melting front is likely on the order of the rate with which heat is conducted through the film, and thus the melt interface does not "feel" the presence of the decrease in barrier layer thickness. It should also be noted that the $\mathrm{x}$-ray peak may be indexed as the martensitic (101) orientation. Again, this strong texture suggests that nucleation may have occurred heterogeneously, although the presence of the preferred texture within the partial melting regime provides motivation for further investigation. Furthermore, the presence of martensite at room temperature suggests that the martensitic phase transformation temperatures are thus above room temperature and this system would be suitable for applications requiring the shape memory effect.

\section{Conclusion}

It has been shown that $1 \mu \mathrm{m}$, amorphous, equiatomic NiTi films may be crystallized via pulsed laser crystallization techniques. However, it is necessary to provide an absorption enhancing surface layer in order to effectively couple laser energy to the film. Grain size and surface melt duration have been shown to increase monotonically as a function of energy density while surface texture remained unvarying for films deposited on 200 and $500 \mathrm{~nm}$ silicon nitride barrier layers. Furthermore, it has been shown that the barrier layer thickness plays an important role in controlling resultant grain size with substantially larger grains obtainable for films on 500 
$\mathrm{nm}$ of silicon nitride. Further investigation is required on two fronts. The first is further determining the nature of the nucleation and growth process. The second is using the knowledge of microstructure control gained by this investigation and examining the resulting shape memory properties of the film.

\section{Acknowledgement}

This work acknowledges the Columbia University MRSEC supported by NSF DMR-0213574, and NSF grants under GK12-0338329 and DMI0355432 and DMR-0347095 (Yale). This work also acknowledges Dr. Cevdet Noyan for his assistance with x-ray diffraction analysis.

\section{References:}

[1] Simidzu, H. , Katayama, S. \& Mastunawa, A. (1991), "Laser Rapid Solidification Microstructure of Single Crystal Aluminum Alloys", Proceedings of the Laser Materials Processing - ICALEO '90, 492-501.

[2] Lin, C.J. \& Spaepen, F. (1986), "Nickel-Niobium Alloys Obtained by Picosecond Pulsed Laser Quenching”, Acta Metallurgica, v 34, n 7, p 1367-1375.

[3] Sposili, R., Crowder, M. \& Im, J.S. (1997) "SingleCrystal Si Films Via a Low-Substrate-Temperature Excimer-Laser Crystallization Method", MRS Proceedings, v 452, Advances in Microcrystalline and Nanocrystalline Semiconductors, p 953-958.

[4] Im, J. S., Crowder, M. A., Sposili, R. S., Leonard, J. P., Kim, H. J., Yoon, J. H. Gupta, V. V., Jin Song, H. \& Cho, H. S. (1998) "Controlled Super-Lateral Growth of Si Films for Microstructural Manipulation and Optimization”, phys. stat. sol. (a) 166, 603-617.

[5] J.P. Leonard, "Nucleation Rate in the Si-SiO2 Thin Film System", Doctoral Thesis, Columbia University, 2000.

[6] Lin, C.-J., Spaepen, F. \& Turnbull, D. (1983), "Picosecond Pulsed Laser-Induced Melting and Glass Formation in Metals", Journal of Non-Crystalline Solids, v 61-62, n pt 2, Jan, p 767-772.

[7] Lin, C.-J. \& Spaepen, F. (1984), "Metallic Glasses and Metastable Crystalline Phases Produced by Picosecond Pulsed Laser Quenching”, Materials Research Society Symposia Proceedings, v 28, p 75-80.

[8] Spaepen, F. (1986), “Thermodynamics and Kinetics of Metallic Alloy Formation by Picosecond
Pulsed Laser Irradiation", High Temperature Materials and Processes, v 7, n 2-3, p 91-100.

[9] Hau-Riege, C.S., Hau-Riege, S.P. \& Thompson, C.V. (2001), "Simulation of Microstructural Evolution Induced by Scanned Laser Annealing of Metallic Interconnects", Journal of Electronic Materials, v 30, n 1, p 11-16.

[10] Ishida, A. \& Sato, M. (2003), "Thickness effect on shape memory behavior of Ti-50.0at.\%Ni thin film", Acta Materialia, 51, 5571-5578.

[11] Ishida, A., Takei, A., Sato, M. \& Miyazaki, S. (1995), "Shape Memory Behavior of Ti-Ni Thin Films Annealed at Various Temperatures", Materials Research Society Symposia Proceedings, v 360, , p 381-386.

[12] Ishida, A., Takei, A., Sato, M. \& Miyazaki, S. (1996), "Stress-Strain Curves of Sputtered Thin Films of Ti-Ni”, Thin Film Solids, 281-282, 337-339.

[13] Gil, F.J., Manero, J.M. \& Planell, J.A. (1995), "Effect of Grain Size on the Martensitic Transformation in NiTi Alloy", Journal of Material Science, 30, 2526-2530.

[14] Lee, H.-J., Ni, H., Wu, D. T., \& Ramirez, A.G. (2005), "Grain size estimations from the direct measurement of nucleation and growth" Applied Physics Letters 87, 124102.

[15] Lee, H.-J. \& Ramirez, A.G. (2004), "Crystallization and Phase Transformations in Amorphous NiTi Thin Films for Microelectromechanical Systems", Applied Physics Letters, Vol. 85, No. 7, 1146-1148.

[16] Makino, E., Mitsuya, T. \& Shibata, T. (2000), "Dynamic actuation properties of TiNi shape memory diaphragm", Sensors and Actuators 79, 128-135.

[17] Wibowo, E., Kwok, C. Y. \& Lovell, N. H. (2004) "Two-way Actuation of Bi-layer Cantilever of Nickel Titanium and Silicon Nitride Thin Films by Shape Memory Effect and Stress Relaxation", Device and Process Technologies for MEMS, Microelectronics, and Photonics III, Proceedings of SPIE Vol. 5276.

[18] Selden, B., Cho, K. \& Asada, H. (2006), "Segmented shape memory alloy actuators using hysteresis loop control", Smart Materials and Structures, 15, 642-652.

[19] Makino, E., Mitsuya, T. \& Shibata, T. (2000), "Micromachining of TiNi Shape Memory Thin Film 
for Fabrication of Micropump", Sensors and Actuators, 79, 251-259.

[20] Wang, X., Bellouard, Y. \& Vlassak, J.J. (2005), "Laser Annealing of Amorphous NiTi Shape Memory Alloy Thin Films to Locally Induce Shape Memory Properties", Acta Materialia, 53, 4955-4961.

[21] Bellouard, Y., Lehnert, T., Bidaux, J.-E., Sidler, T., Clavel, R. \& Gotthardt, R. (1999), "Local annealing of complex mechanical devices: a new approach for developing monolithic micro-devices", Materials Science and Engineering A, 795-798.

[22] He, Q., Hong, M.H., Huang, W. M., Chong, T. C., Fu, Y. Q. \& Du, H. J. (2004), "CO2 laser annealing of sputtering deposited NiTi shape memory thin films", Journal of Micromechanics and Microengineering, 14, 950-956.

[23] Porter, D.A. \& Easterling, K.E. "Phase Transformations in Metals and Alloys", 1981, Van Nostrand Rheinhold, Berkshire, England.

[24] Aziz, M.J. (1982), "Model for Solute Redistribution During Rapid Solidification", Journal of Applied Physics, 53(2), 1158-1168.

[25] Khelfaoui, F., Bellouard, Y., Gessmann, T., Wang, X., Vlassak, J. \& Hafez, M. "An Investigation of the Oxidation of Laser and Furnace-Annealed SputterDeposited NiTi Thin Films Using Reflectivity Measurements", SMST 2004, Baden-Baden, Germany.

[26] Sposili, R. "Crystalline Silicon Thin Films for Thin-Film Transistor Applications Via Excimer Laser Irradiation", Doctoral Thesis, Columbia University, 2001.

\section{Meet the Authors}

Andrew J. Birnbaum received his B.S and M.S. from Carnegie Mellon University. He is currently a doctoral student at the Manufacturing Research Laboratory at Columbia University.

Xu Huang is a Postdoctoral Associate in Department of Mechanical Engineering at Yale University. His research interests are in shape memory alloys and electronic packaging. He received his doctorate degree from Nanyang Technological University, Singapore, master's degree from University of Science and Technology Beijing, and bachelor's degree from Southern Institute of Metallurgy, P.R. China, all in materials science and engineering.
Ainissa G. Ramirez is an Associate Professor of Mechanical Engineering at Yale University. Prior to her appointment at Yale, she was a member of technical staff at Bell Laboratories, Lucent Technologies in Murray Hill, NJ. Her research interests are in thin film shape memory alloys and universal solders. She received her doctorate and master's degrees from Stanford University, and bachelor's degree from Brown University, all in materials science and engineering.

Dr. Ui-Jin Chung received his B.S., M.S. and PhD from Seoul National University in Material Science and Engineering. He previously worked as a senior engineer at Samsung Electronics and is currently an associate research scientist in the laser crystallization lab at the Applied Physics and Applied Math Department, Columbia University.

Sean Polvino received his Sc.B. from Brown and M.S. from Columbia University. He is currently a doctoral student in the Applied Physics and Applied Math Department at Columbia University.

Dr. Y. Lawrence Yao is currently department chair of Columbia University's Mechanical Engineering Department and director of the Manufacturing Research Laboratory. He received his Ph.D. from the University of Wisconsin-Madison in 1988. He also serves on the Board of Directors of LIA. 